\title{
Evolution of the Web: from Web 1.0 to 4.0
}

\author{
Asaad Khaleel Ibrahim \\ Information Technology \\ Duhok Polytechnic University \\ Duhok - Iraq \\ Asaad.khaleel@dpu.edu.krd \\ https://doi.org/10.48161/qaj.v1n3a75
}

\begin{abstract}
The internet has become a vital component of the twenty-first century as technology has advanced. The number of new technologies emerging in tandem with the qualities supplied by the Internet is rapidly increasing. The World Wide Web (WWW), which is commonly referred to as the world's largest information environment, is a vital virtual environment in which internet users may trade, read, and publish information using a Web browser. Web 1.0, Web 2.0, and Web 3.0 technologies have all been seen and are still being observed in this review paper. However, there is no clear definition for Web 4.0, which is a 4th generation web technology, in the literature. Web 4.0 has multiple dimensions, as seen by the first examples that have appeared. Big data, augmented reality, machine-to-machine communication (M2M), cloud computing, and artificial intelligence (AI) technologies, as well as smart agents, will be able to integrate in the future years. Web 4.0 is a web technology revolution that includes a new internet of things (IoT) that interacts with a variety of models. The goal of this study is to clarify the notion of Web 4.0, which is viewed as an intelligent and symbiotic (human-machine interaction) network with massive interfaces and linkages, as well as to contribute to the literature by studying its many dimensions and investigating its links with new generation technologies.
\end{abstract}

Keywords: Web Technologies, semantic web, Web 4.0, Symbiotic Web.

\section{INTRODUCTION}

The Internet offers great conveniences to people in developing ageappropriate innovations and providing connections with constantly updated technologies [1]. In the last 20 years, there has been a lot of progress in web technologies with the use of the features offered by internet connections [2]. Looking at the current development process since the development of web technology, each period has emerged with new features and opportunities. The formation process of these developments is changing day by day with the change of the relationship between the concept of the internet and the user.[3].
In 1989, while working at CERN, British scientist Tim Berners-Lee devised the World Wide Web. Initially, the Web was designed and designed to address the needs of scientists from colleges and institutions around the world for automated information sharing. [4]. This technology has been developed based on the detailed and easy access of internet users to content in large databases over the Web. In this process, Social computing should occur on the Web and a new era called social machines should emerge [5]. It was also argued that "the development of interactive new Web applications", which analyzes large amounts of data that people are trying to do with increasing technological capabilities [6].

Web 1.0 emerged as the first learning network in the development of web pages with HTML, which determines the emergence of a new Web paradigm. It is then actively used as an interaction and communication network in Web 2.0 and Web 3.0 as an integration network [2]. Web 4.0, which is integrated with various technologies and is considered as the ultra-intelligent Web, has emerged as the fourth generation Web technology. Although there is no definitive judgment about Web 4.0 technology or a specific definition of how it will work in the literature, thoughts about how this technology will work are quite common. Various definitions related to this concept have been made in the literature. It states that "social machines" will have the mission of organizing our social processes invisibly. In this sense, it is thought that greater cooperation is needed between researchers in the field of the web and AI [7 - 9].

Web 4.0, which will be in an important position between 2020 and 2030, is associated with four technologies such as artificial intelligence, nanotechnology, telecommunications and controlled interfaces, as well as the concept of the IoT [10]. The IoT supported living, e-health, advanced learning, retail, automation, financial and industrial production, logistics, business process management and intelligent transportation can be listed as some of its potential benefits [11-14]. It is also possible that there will be a technology 
that provides more interaction between humans and machines in line with the technology and studies carried out in the information age. It is thought of as a qualified and interactive web technology where people can communicate directly with machines, shape the world and respond to computers [15 - 16].

With the emergence of Web 4.0 as a new Web generation, Web 4.0 will combine all the features of Web 2.0 and Web 3.0 and is seen as a technology that can be found anywhere [16]. It is said that computer users will have a powerful technology that intelligently conducts and interacts with the web environment Thinks to the powerful and intelligent interfaces of this technology. With the increasing communication and information channels developing day by day, it is an important element for the Web world to develop qualities that can solve these needs. Therefore, Web 4.0 is expected to be an environment that can collaborate in industry, political, social and other important communities by providing access to important audiences with the simultaneous networking of technology, and participation in online networks that provide global transparency, governance and distribution systems [17 - 18]. In this context, Web 4.0 is classified as a symbiotic (human-machine interaction) web where data interactions occur between humans and smart devices and the IoT [19].

Within the scope of this study, a literature review of Web 4.0 with its intelligent and highly interactive features, which is the next generation of web technology, was given and comments on the innovations and features it will present to the Web world in the future are included. It is also aimed to analyze the dimensions of this technology by clarifying quite different approaches about what is or maybe about the concept of Web 4.0.

\section{WEB TECHNOLOGIES}

Machine and computer algorithms grew quickly towards the latter of the 20th century and are a technological revolution, are involved in different ways in every aspect of the current era [20]. Today, AI technologies, which are increasing in importance, were introduced for the first time by Alan Turing as the concept of machine intelligence, in other words, 'Thinking machines'. In 1950, a test called the Turing Test took a big step by measuring a machine's ability to behave intelligently equivalent to or similar to a human [21].

In the formation of the intelligent web platform, web technology is needed, which has the ability to read, understand and give feedback on the data obtained from the machine language. Because of the increasingly large amount of data and the need to be converted. Therefore, the 4th generation of the Web is expected to experience a Web page integrated with AI algorithms [22].
The data obtained from the Web will be converted into meaningful and processed data by reasoning. With the establishment of smarter Web environments, the features offered by AI technology will be used to facilitate Web mining. Intelligent web revolution 4.0, in which various opinions and ideas are proposed, is based on the development and use of features implemented by advances in AI and its associated Web 3.0. With a Web environment where objects interact with each other through the virtual world, Web 4.0 is a technology position consisting of the integration of Big data, M2M communication, AI, Cloud Computing, Augmented reality (VR), Intelligent agents, and IoT technologies [23].

\subsection{Web 1.0}

The Web 1.0 period, known as the first period of the Internet, is a structure that contains static HTML pages created in 1995 for easy access when the research was conducted on the Web. In this structure, users were passively located on a web network without interacting [24]. Internet users were also not authorized to add or comment on any content. Users only used their web media for information. Web 1.0 was basically a static, one-way network that was read-only and focused solely on learning information. However, with the day-to-day development of technology, this Web network has been replaced by Web 2.0 technologies. Figure 1 shows the connection direction of Web 1.0 technology.

\section{Web 1.0}

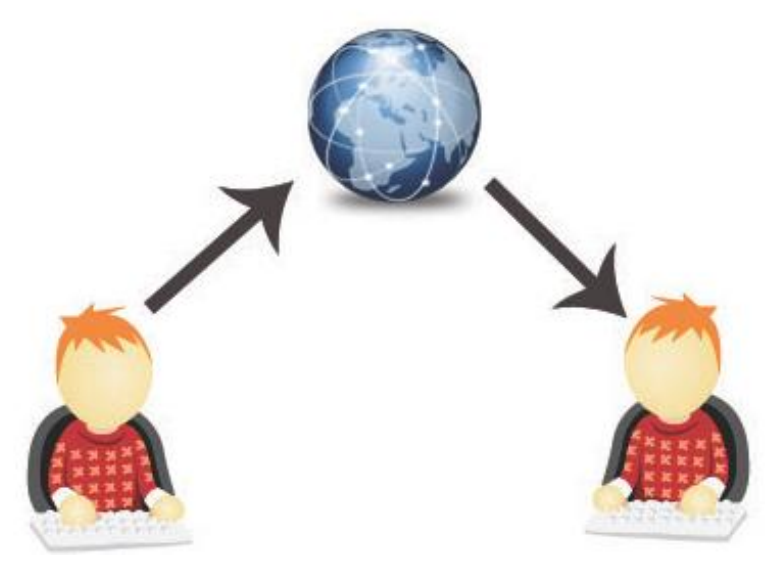

Figure 1. Web 1.0 one-way connectivity platform [25]

\subsection{Web 2.0}

The term Web 2.0 was first used in 2004 by Dale Dougherty, who was O'Reilly's vice president, at a conference with MediaLive. Web 2.0 explains why you should move to the internet In the computer industry, the Web is defined as becoming an interaction so that more 
people can create applications using the platforms offered by the network [26]. The second generation, known as Web 2.0 was coined in 2004 when the Web became a popular medium. Web 2.0 has created a user-centric and collaborative environment [27].

A dynamic and two-way connectivity platform has occurred, with high engagement, user participation in content production, and sharing. During this period, with the emergence of very popular Web sites such as Wikis, YouTube, Facebook, Twitter, Blogs and Instagram, a serious flow of information began to occur on social networks [28]. Websites have become interactive, providing feedback among users. With these developments in the web world, mobile access connectivity has also grown significantly. With the launch of smartphones, users have moved to easier environments where they can interact with each other. Thus, the use of web 2.0 environments has increased greatly. Internet users have been part of a platform where various shares such as photos, files, music, videos are made using various web tools. Using the XML (Extensible Markup Language) tag language, the backbone of the internet, an independent platform in the infrastructure of Web 2.0, provides information flow with permissions from databases on the Web. With the RSS (Really Simple Syndication/ Rich Site Summary) Web page declarator, it is possible to query the data contained in different systems and information flows that are active with various web resources such as XML. Web 2.0 is also known as a user-centred, highly interactive read-write web network. Web 2.0 users have dropped most of the controls they are accustomed to in Web 1.0 and moved to the platform where they interact more with other users [29]. Figure 2 shows the connectivity aspects of Web 2.0 technology.

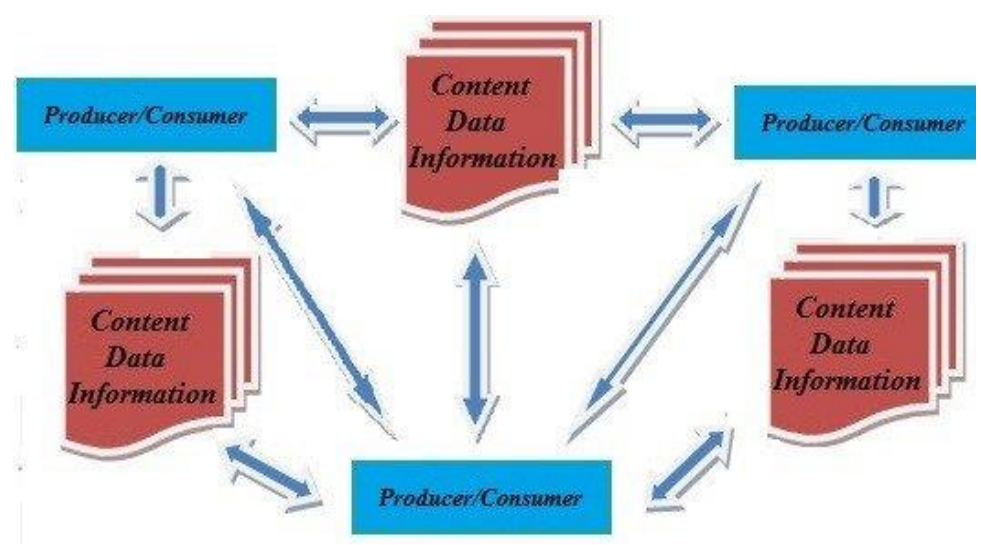

Figure 2. Web 2.0 two-way connectivity platform [30]

\subsection{Web 3.0}

The (WWW) Consortium (W3C) began revolutionary work on Web 3.0 in 2006 [31]. From 2010 until the present, the third generation of the Web, also known as Web 3.0 or Semantic Web, has existed [32-34]. Web 3.0 aims to regulate how content is searched and displayed by the user. With the arrival of RDF and OWL (Web Ontology Language) languages with this technology, a Web environment based on the information obtained from web pages and content owned by users was prepared [35]. It has been transformed into a platform that manages data based on the evaluation of user data obtained from search engines and inferences made. The basis of the semantic network, inspired by XML, has been a novelty of Web 3.0 in making sense of OWL language data [36-38].

The goal is to customize and optimize online search based on users' history, interests, and desires. Web 3.0 ensures that all information and data on the web is associated with descriptions. The new web technology is aimed at intertwining content and keywords (metadata) [39]. Web 3.0 is also called the smart Web by many authors because its functions extend beyond traditional search services. The behaviours that Internet users search over the Web can be sorted and customized according to their preferences. Web 3.0's basic idea is to identify build data and enable it to connect with each other to be more effective, supporting the creation of user profiles [40].

In order to provide new information flows, it integrates and analyzes the data obtained from various datasets into its content, improves data management, increases users' satisfaction and ensures cooperation on the social network. With Web 3.0, significant progress has been made in the formation of big data that many institutions want to have today, in increasing customer satisfaction and in collecting data to meet their expectations. With the data in the data warehouses created in line with the searches of users with Web technologies, many institutions benefit from the opportunities offered by this technology in their decision-making stages. Web 3.0 was passed in the analysis of big data where AI and fuzzy logic are combined [41].

Table1: Comparison in between Web1.0, Web 2.0 and Web3.0

\begin{tabular}{|l|l|l|l|}
\hline Parameter & Web 1.0 & Web 2.0 & Web 3.0 \\
\hline Year of foundation & 1994 & 2004 & 2006 \\
\hline Coined By & Tim Berners- Lee & Dale Dougherty & John Markoff \\
\hline
\end{tabular}




\begin{tabular}{|l|l|l|l|}
\hline Generation & Cognition & Communication & Co-operation \\
\hline Known As & $\begin{array}{l}\text { "The Web" or "The } \\
\text { Internet" }\end{array}$ & "Web of Documents" & "Web of Data" \\
\hline Human Interaction & Not applicable & Not applicable & Personalized \\
\hline Semantics & Relational Schema & XSD/DTD & Applied Using RDFS/OWL \\
\hline Metadata & Simple SQL & SQL along XPath & $\begin{array}{l}\text { SPARQL: Protocol and RDF } \\
\text { Query Language }\end{array}$ \\
\hline Query Language & Not applicable & Not applicable & Applied \\
\hline Machine Learning & Relational & Relational Hierarchical & Graph \\
\hline Data Model & Hyperlink & Hyperlink & URI \\
\hline Linking & To search the data & to share the data with others & $\begin{array}{l}\text { to search, Integrate and } \\
\text { control data }\end{array}$ \\
\hline Used for & HTML & HTML XHTML, XML & RDF, RDF a, Micro formats \\
\hline Data Representation & &
\end{tabular}

\section{. WEB 4.0 AND DIMENSIONS}

Web 4.0 may be divided into three categories: ultra-intelligent electronic agents, symbiotic webs, and ubiquitous webs, it is quite curious to see how effective its quality and performance will be. However, with Web 3.0, great progress is being made, and there is a lot of consensuses that Web 4.0 will make it easier to make decisions by making smart interfaces and being smart when reading Web content. There are opinions that it will be characterized as WebOS (a Linux-based mobile operating system). In particular, it is said that the concept of Industry 4.0, which first appeared in Germany in 2011, will be associated with Web 4.0. Smart factories, one of the objectives of Industry 4.0 technology, are also thought to be associated with the IoT in interaction. Cybersystems, Web services and the IoT are very important to produce more efficiently in this process and to make these productions customizable. In that context. There is the development of connections with Web 4.0. For example, if you want to use Consider the analysis of machine processes that are in communication with each other in a smart factory by collecting them in a database and analyzing them on the web. It is not a distant idea that the ultra-intelligent Web feature plays an active role in the processed data obtained and that Web technology can be directed at these objects during the decision-making stages [42].

In recent years, the analysis of information obtained with big data has gained a lot of momentum in customer relationship management (CRM) in the creation of customer network profiles of institutions. The underlying idea of the creation of these customer portfolios is the idea that the IoT will actively play a role in the creation of marketing strategies of organizations and the conversion of customers' purchasing characteristics into meaningful data. Organizations strive to create a platform where their large data can be easily analyzed with the support of selflearning, content-generating smart agents and sensors in expanding the data they obtain from these profiles. For example, imagine a customer entering the store and suddenly getting a message on their phone based on information obtained by smart agents. The message may be able to contain information about the product researches that the customer has done with his phone and which aisle should be directed to the relationship with the products he purchases. With the intelligent interfaces developed, the future position of machine intelligence can be considered in this way. Web 4.0 technology, which is foreseen to have creative features with the algorithms developed, is also expected to have human and machine intelligence is expected to become a common field. The Smart Web feature, where interconnected devices are created and big data analytics is performed, is thought to be a centre for human-machine interaction designed with various software agents [43].

for instance, it is required to be registered via the web to attend the conference. When a hotel is booked for your destination city, a page might be found where your hotel reservation could be made based on your old data when it was registered for you, 
contains data and documents learned by the web machines. With web 4.0, it is seen that for personalized agents with AI, a platform will be there in the machine-machine and machine-human interaction cycle where cloud technologies and systems can be managed anywhere regardless of location [44]. The concept of web 4.0 is associated with smart agents, mobile technologies, and cloud computing technologies, which is now used in a broadcast way. In recent years, cloud computing has also attracted the attention of industrial companies and academia organizations [45 - 46].

At the heart of the Cloud lies the fact that data and applications are accessible from any device connected to the Internet anytime, anywhere [47]. This feature of the cloud eliminates both the cost and complexity associated with the integration and maintenance of an information infrastructure. With these features, Web 4.0 will be able to achieve great efficiency in the business world with the increase of successful business models as a result of its integration with Cloud computing [48]. Depending on cloud computing technology, which is the data storage area that internet users access regardless of location and place, examples of the development of environments that do not require hard drives and can work on the Web with AI are seen. Looking at the present as examples of Web 4.0, we can say that Cortana (Android), Siri (iPhone), Google Docs, YouOS, DesktopTWo applications are examples of this technology [49], today's hugely popular machine learning application community, offers free GPU support and any software With the support of the editor, the program is provided with a customizable environment where data analysis is performed by accessing the large data and code pool published by the community. These transactions with Google Cloud, which is used to perform transactions, It is seen as a product of 4.0 technology. In this context, it can be shown as an example that makes it very easy for users to perform their transactions without using physical disk usage using the features offered by cloud computing technology.

With the development of 3D browsers, it may be possible to browse the desired stores virtually via the Web. If it is decided to buy a product, a platform can be experienced by selecting cheaper products with the web assistant. With Web 4.0, devices can communicate with each other to process information and act in the Web environment. With less human intervention, the desired transactions can be performed after processing the devices with the data received from the Web. Web 4.0 can help eliminate the boundary between humans and machines, give machines the ability to think like humans and take on tasks that people have a hard time with. In this direction, time losses can be avoided and more resources can be saved. With applications that improve by connecting smart devices to the internet, people can also have personal machines suitable for their lifestyle. In addition, better decision-making, Web 4.0, can be proposed as a solution to the problems that arise rather than computers simply generating information. Thus, it will be possible for institutions to make decisions effectively when they cannot overcome big data [50]. In line with AI applications, it is possible to make decisions that managers have difficulty making easily. In this process, applications consisting of the integration of AI and Web 4.0 will inevitably be seen soon. Today, institutions expect fewer costs and more labour. Therefore, less manpower and more machine thinking can be adopted thanks to the applications developed for the lower management levels in the institutions[42].

Table 2: Key Feature and used techniques of Web4.0

\begin{tabular}{|l|l|}
\hline Feature & Technology \\
\hline Virtual assistance as 2D/3D Experts system & Experts system \\
\hline Accuracy and analysis of data & Data sciences \\
\hline Security & Blockchain \\
\hline Decision making and precision & Adaptive fuzzy-based inferencing \\
\hline Optimization & Machine learning \\
\hline Live human-like assistance with visualization & Augmented reality \\
\hline Human interaction/natural language understanding & NLP \\
\hline
\end{tabular}

Looking at some of the challenges that Web 4.0 can bring with various applications, there are systemic problems caused by internet density and excess connectivity with this technology that users will actively use. One of the most critical developments of the web is probably that the intensity of its online transactions affects daily life. Because physically and virtually, the challenges 
that objects connected to someone can create when there is a connectivity problem are possible. For example, the phone you lost think of it as searching the Web to find your phone. When you encounter a connection problem as a result of a systemic problem, you may experience the negativity of not having control. One of the downsides that come with Web 4.0 in the future is that wireless connections and actual telecommunications lines will only need a language that should be of the same standards by all devices considering that they belong to a particular company [51].

Considering the companies that are competing in the global world, it seems very difficult to establish connections with a certain standard. In addition, in this age of threat to information security, it may be difficult to access personal data and securely store information by third parties if the confidentiality and reliability of databases created by internet users are not guaranteed. Today, the natural language processing (NLP) technique, which has examples such as Google translation, is expected to advance and increase with the innovations that come with Web 4.0 as the new communication interface model between man and machine [52]. However, it is more prominent that technologies such as social networks, IoT, big data, AI and M2M play a key role in the adoption and implementation of Web 4.0 [53].

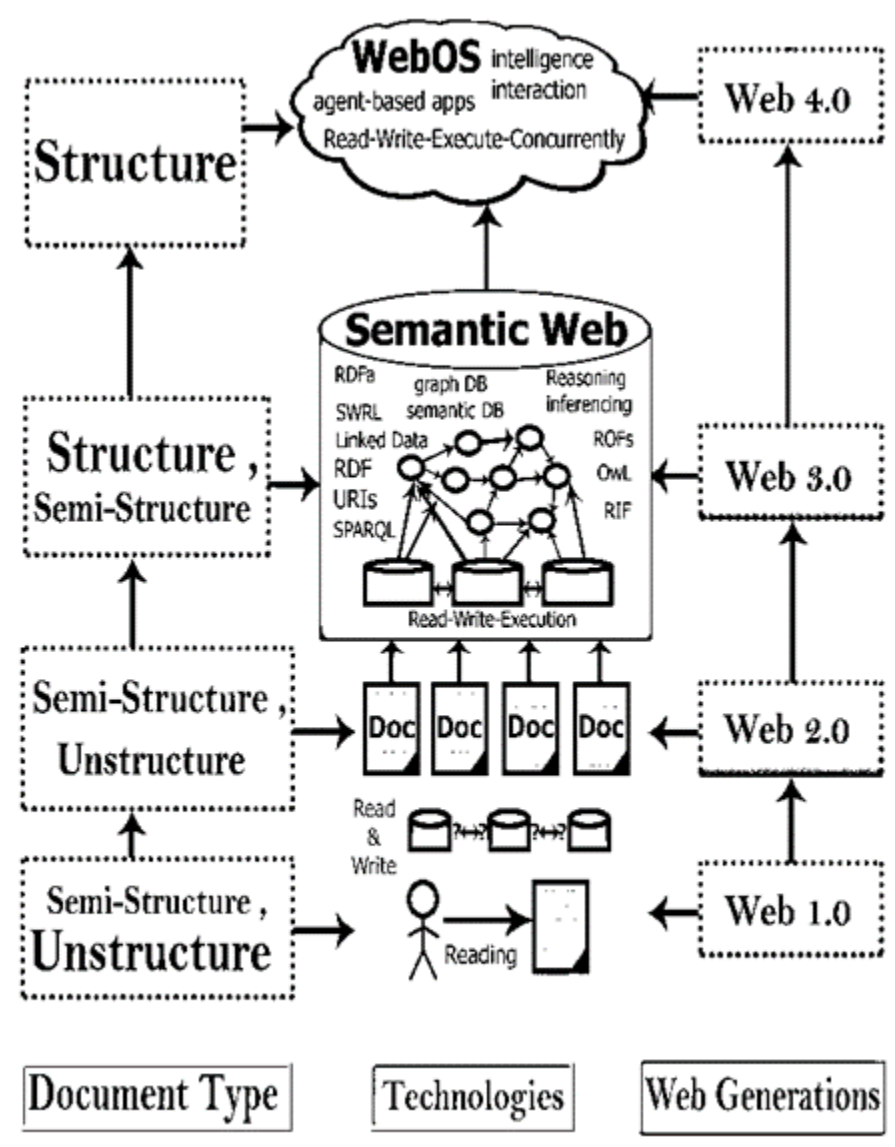

Figure 3. shows Web technologies and document types [30].

With the development of applications and examples starting with Web 1.0, the journey to Web 4.0 is on its way to becoming an ultra-smart web that facilitates the operations of internet users with updates and smartening of web technology at every step. With these developments and the connections offered by technology, worldwide comprehensibleness in the field of industry, political, social and different networks, it seems that there will be a technology that holds together governance, dissemination, interest and cooperation between key networks [54].

\section{CONCLUSION}

The web environment is constantly evolving in a borderless way. In the last decade, various definitions of Web 4.0 have been made by many authors when looking at the literature. However, there is no definitive definition of Web 4.0 in relation to previous previously accepted definitions of Web 1.0, Web 2.0, and Web 3.0. In recent years, the internet has become even more widespread 
in an age when wireless networks are increasingly actively using smartphones and tablets throughout the day. Looking at the connection of technologies to Web 4.0, it is seen that important stages will be made and they will play a key role in big data analysis with Web 4.0, the next stage of Web technologies. With the applications offered by AI technology, it is seen that users need new technologies to have more interactive and immersive experiences.

Web 4.0 seems possible to connect all devices in a real and virtual world in real-time, such as services, virtual reality services, and natural language services where intelligent agents and sensors interact. Another dimension is that web pages, web applications, videos, and photos connected by Web 4.0 can be interactive. In this process, the good configuration of information is of great importance. In this context, it is thought that access to the Internet can be made not only by humans but also from other physical objects, devices and tools arising from the concept of the IoT. With the desired new technology elements, the concept of Web 4.0 appears as a new paradigm. With Web 4.0, it is thought that an interactive environment with virtual computer environments where AI, operating systems and cloud computing technology will be activated and published very soon. Web 4.0, which looks like it will take an active role by 2020 , is proof that this technology will go further by the beginning of integration into today's technologies. In this age of ever-increasing internet users, Web 4.0 is moving towards becoming an ultra-intelligent and intelligent web technology, which can be connected to the next generation of technologies using websites, blogs, online publications, social networks, databases and more interaction networks. For this reason, it is possible to associate the concept of Web 4.0 with various technologies.

There is a possibility of many developments regarding Web 4.0, positive and negative. However, in the technology-oriented global network, web technologies appear to be at the heart of society and have made great progress in technology, while web generations and it is quite normal for the stages to develop in this direction. When a perspective is developed for the future, it is a fact that Web 4.0 will be created with new updates to the innovations introduced by Web 3.0. Therefore, the Web that is expected to come later In addition to ultra-intelligent robots, machines and other technologies developed with the update of the next-generation technologies introduced by Web 4.0 technology, the arrival of machines that manage to think emotionally with sensuality, which is unique to humans, does not seem far off.

\section{Reference}

[1] K. J. A Zeebaree SRM Zeebaree, "Designing an Ontology of E-learning system for Duhok Polytechnic University Using Protégé OWL Tool,' J Adv Res Dyn Control Syst Vol, vol. 11, no. 5, pp. 24-37, 2019.

[2] K. Jacksi and S. M. Abass, "Development history of the world wide web," Int J Sci Technol Res, vol. 8, no. 9, pp. 75-79, 2019.

[3] S. Zeebaree, R. R. Zebari, and K. Jacksi, "Performance analysis of IIS10. 0 and Apache2 Cluster-based Web Servers under SYN DDoS Attack," TEST Eng. Manag., vol. 83, pp. 5854-5863, 2020.

[4] K. Jacksi, N. Dimililer, and S. R. M. Zeebaree, "A Survey of Exploratory Search Systems Based on LOD

Resources," in PROCEEDINGS OF THE 5TH INTERNATIONAL CONFERENCE ON COMPUTING \& INFORMATICS, COLL ARTS \& SCI, INFOR TECHNOL BLDG, SINTOK, KEDAH 06010, MALAYSIA, 2015, pp. 501-509.

[5] K. Jacksi, S. Zeebaree, and N. Dimililer, "Design and Implementation of LOD Explorer: A LOD Exploration and Visualization Model,” J. Appl. Sci. Technol. Trends, vol. 1, no. 2, pp. 31-39, 2020.

[6] T. Berners-Lee, "The World Wide Web: A very short personal history," Tim Bern.-Lee, vol. 7, 1998.

[7] N. Shadbolt, M. Van Kleek, and R. Binns, "The rise of social machines: the development of a human/digital ecosystem," IEEE Consum. Electron. Mag., vol. 5, no. 2, pp. 106-111, 2016.

[8] R. Ibrahim, S. R. M. Zeebaree, and K. Jacksi, "Semantic Similarity for Document Clustering using TFIDF and Kmean," Master's Thesis, University of Zakho, Zakho, 2020.

[9] R. Ibrahim, S. Zeebaree, and K. Jacksi, "Survey on Semantic Similarity Based on Document Clustering," Adv Sci Technol Eng Syst J, vol. 4, no. 5, pp. 115-122, 2019.

[10] M. A. Sadeeq, S. R. Zeebaree, R. Qashi, S. H. Ahmed, and K. Jacksi, "Internet of Things security: a survey," 2018, pp. 162-166.

[11] M. Parvathi and R. Mariselvi, "A bird's eye on the evolution-Web 1.0 to Web 5.0: Lib 1.0 to Lib 5.0," Int. J. Adv. Res. Trends Eng. Technol., vol. 4, no. 4, pp. 167-76, 2017.

[12] R. R. Zebari, S. Zeebaree, K. Jacksi, and H. M. Shukur, "E-business requirements for flexibility and implementation enterprise system: A review," Int. J. Sci. Technol. Res., vol. 8, no. 11, pp. 655-660, 2019.

[13] K. Jacksi, M. A. Sulaiman, and R. H. Saeed, "The Importance of E-Learning in the Teaching Processor 
Secondary Schools/Review Article," Acad. J. Nawroz Univ., vol. 10, no. 1, pp. 53-62, 2021.

[14] K. Jacksi, F. Ibrahim, and S. Ali, "Student Attendance Management System," Sch. J. Eng. Technol. SJET, vol. 6, no. 2, pp. 49-53, 2018.

[15] B. Khoo, "RFID-from Tracking to the Internet of Things: A Review of Developments," in 2010 IEEE/ACM Int'1 Conference on Green Computing and Communications \& Int'l Conference on Cyber, Physical and Social Computing, 2010, pp. 533-538.

[16] F. Almeida, "Concept and dimensions of web 4.0," Int. J. Comput. Technol., vol. 16, no. 7, 2017.

[17] S. Aghaei, M. A. Nematbakhsh, and H. K. Farsani, "Evolution of the world wide web: From WEB 1.0 TO WEB 4.0," Int. J. Web Semantic Technol., vol. 3, no. 1, pp. 1-10, 2012.

[18] L. M. Haji, S. Zeebaree, O. M. Ahmed, A. B. Sallow, K. Jacksi, and R. R. Zeabri, "Dynamic resource allocation for distributed systems and cloud computing," TEST Eng. Manag., vol. 83, pp. 22417-22426, 2020.

[19] L. Atzori, A. Iera, and G. Morabito, "The internet of things: A survey," Comput. Netw., vol. 54, no. 15, pp. 2787-2805, 2010.

[20] A. Kordon, Applying computational intelligence: how to create value. Springer Science \& Business Media, 2009.

[21] J. Howard, "Artificial intelligence: Implications for the future of work," Am. J. Ind. Med., vol. 62, no. 11, pp. 917-926, 2019.

[22] D. Raggett, "The web of things: Challenges and opportunities," Computer, vol. 48, no. 5, pp. 26-32, 2015.

[23] P. Y. Abdullah, S. R. Zeebaree, H. M. Shukur, and K. Jacksi, "HRM system using cloud computing for Small and Medium Enterprises (SMEs)," Technol. Rep. Kansai Univ., vol. 62, no. 04, p. 04, 2020.

[24] M. Tekdal, S. Sayginer, and F. Baz, "Developments Of Web Technologies And Their Reflections To Education: A Comparative Study,” Educ. Instr. Stud. World, vol. 8, no. 1, pp. 17-27, 2018.

[25] "Web 1.0," The Business of Social. https://thebusinessofsocial.wordpress.com/tag/web-1-0/ (accessed Jun. 05, 2021).

[26] M. Needleman, "Web 2.0/Lib 2.0-What Is It? (If It's Anything at All)," Ser. Rev., vol. 33, no. 3, pp. 202-203, Sep. 2007, doi: 10.1016/j.serrev.2007.05.001.

[27] K. Jacksi, R. Kh. Ibrahim, S. R. M. Zeebaree, R. R. Zebari, and M. A. M. Sadeeq, "Clustering Documents based on Semantic Similarity using HAC and K-Mean Algorithms," in 2020 International Conference on Advanced Science and Engineering (ICOASE), Dec. 2020, pp. 205-210. doi: 10.1109/ICOASE51841.2020.9436570.

[28] S. R. M. Z. Adel AL-Zebari Karwan Jacksi and Ali Selamat, "ELMS-DPU Ontology Visualization with Protégé VOWL and Web VOWL," J. Adv. Res. Dyn. Control Syst., vol. 11, no. 1, pp. 478-485, 2019.

[29] Y.-C. Hsu, Y.-H. Ching, and B. L. Grabowski, "Web 2.0 applications and practices for learning through collaboration," in Handbook of research on educational communications and technology, Springer, 2014, pp. 747758.

[30] K. Nath and R. Iswary, "What comes after Web 3.0? Web 4.0 and the Future," in Proceedings of the International Conference and Communication System (I3CS'15), Shillong, India, 2015, pp. 337-341.

[31] K. Jacksi, N. Dimililer, and S. Zeebaree, "State of the art exploration systems for linked data: a review," Int J Adv Comput Sci Appl IJACSA, vol. 7, no. 11, pp. 155-164, 2016.

[32] N. D. Karwan Jacksi,Subhi R. M. Zeebaree, AN IMPROVED APPROACH FOR INFORMATION RETRIEVAL WITH SEMANTIC-WEB CRAWLING PhD . THESIS. 2016.

[33] S. M. Mohammed, K. Jacksi, and S. Zeebaree, "A state-ofthe-art survey on semantic similarity for document clustering using GloVe and density-based algorithms," Indones. J. Electr. Eng. Comput. Sci., vol. 22, no. 1, pp. 552-562, 2021.

[34] S. M. Mohammed, K. Jacksi, and S. R. M. Zeebaree, "Glove Word Embedding and DBSCAN algorithms for Semantic Document Clustering," in 2020 International Conference on Advanced Science and Engineering (ICOASE), Dec. 2020, pp. 1-6. doi: 10.1109/ICOASE51841.2020.9436540.

[35] S. R. Z. Jacksi, K, N. Dimililer, "State of the Art Exploration Systems for Linked Data: A Review," IJACSA Int. J. Adv. Comput. Sci. Appl., vol. Vol. 7, No, 2016.

[36] A.-Z. Adel, S. Zebari, and K. Jacksi, "Football Ontology Construction using Oriented Programming," J. Appl. Sci. Technol. Trends, vol. 1, no. 1, pp. 24-30, 2020.

[37] N. M. Salih and K. Jacksi, "State of the art document clustering algorithms based on semantic similarity," J. Inform., vol. 14, no. 2, pp. 58-75, 2020.

[38] N. M. Salih and K. Jacksi, "Semantic Document Clustering using K-means algorithm and Ward's Method," in 2020 International Conference on Advanced Science and Engineering (ICOASE), Dec. 2020, pp. 1-6. doi: 10.1109/ICOASE51841.2020.9436588. 
[39] S. Nova, Web 3.0: The Third Generation Web is Coming. 2011.

[40] K. Jacksi, "Design and Implementation of E-Campus Ontology with a Hybrid Software Engineering Methodology,” Sci. J. Univ. Zakho, vol. 7, no. 3, pp. 95100, 2019.

[41] K. Jacksi, S. R., and N. Dimililer, "LOD Explorer: Presenting the Web of Data," Int. J. Adv. Comput. Sci. Appl., vol. 9, no. 1, pp. 45-51, 2018, doi: 10.14569/ijacsa.2018.090107.

[42] F. Almeida, "Concept and dimensions of web 4.0," Int. J. Comput. Technol., vol. 16, no. 7, 2017.

[43] A. J. Jara, A. C. Olivieri, Y. Bocchi, M. Jung, W. Kastner, and A. F. Skarmeta, "Semantic web of things: an analysis of the application semantics for the iot moving towards the iot convergence," Int. J. Web Grid Serv., vol. 10, no. 2-3, pp. 244-272, 2014.

[44] V. Nedeva and S. Dineva, "New learning innovations with Web 4.0," in Proceedings of the 7th International Conference on Virtual Learning (ICVL), Bucharest, Romania, 2012, pp. 316-321.

[45] O. M. Ahmed and W. M. Abduallah, "A Review on Recent Steganography Techniques in Cloud Computing," Acad. J. Nawroz Univ., vol. 6, no. 3, pp. 106-111, 2017.

[46] I. M. Ibrahim et al., "Task scheduling algorithms in cloud computing: A review,” Turk. J. Comput. Math. Educ., vol. 12, no. 4, pp. 1041-1053, 2021.

[47] P. Y. Abdullah, S. R. Zeebaree, K. Jacksi, and R. R. Zeabri, "An hrm system for small and medium enterprises (sme) s based on cloud computing technology," Int. J.

Res.-GRANTHAALAYAH, vol. 8, no. 8, pp. 56-64, 2020.

[48] Z. N. Rashid, S. R. Zebari, K. H. Sharif, and K. Jacksi, "Distributed cloud computing and distributed parallel computing: A review," 2018, pp. 167-172.

[49] H. Kurgun, O. A. Kurgun, and E. Aktaş, "What does web 4.0 promise for tourism ecosystem? A qualitative research on tourism ecosystem stakeholders' awareness," J. Tour. Hosp. Manag., vol. 6, no. 1, pp. 55-65, 2018.

[50] M. J. Singh, "Web 4.0: An intelligent tool from Web-ofthing to Web-of-thought".

[51] N. Choudhury, "World wide web and its journey from web 1.0 to web 4.0," Int. J. Comput. Sci. Inf. Technol., vol. 5, no. 6, pp. 8096-8100, 2014.

[52] D. H. Maulud, S. R. Zeebaree, K. Jacksi, M. A. M. Sadeeq, and K. H. Sharif, "State of art for semantic analysis of natural language processing," Qubahan Acad. J., vol. 1, no. 2, pp. 21-28, 2021.
[53] K. Nath and R. Iswary, "What comes after Web 3.0? Web 4.0 and the Future," in Proceedings of the International Conference and Communication System (I3CS'15), Shillong, India, 2015, pp. 337-341.

[54] C. A. Khanzode and R. Sarode, "Evolution of the world wide web: from web 1.0 to 6.0," Int. J. Digit. Libr. Serv., vol. 6, no. 2, pp. 1-11, 2016. 